\title{
Smart applications to track and record physical activity: implications for obesity treatment
}

This article was published in the following Dove Press journal:

Smart Homecare Technology and TeleHealth

31 July 2014

Number of times this article has been viewed

\section{Siew Sun Wong' \\ Yu Meng' \\ Paul D Loprinzi ${ }^{2}$ \\ Nobuko Hongu ${ }^{3}$}

'School of Biological and Population Health Sciences, Oregon State University, Corvallis, OR, USA; ${ }^{2}$ Department of Exercise Science, Donna and Allan Lansing School of Nursing and Health Sciences, Bellarmine University, Louisville, KY, USA; ${ }^{3}$ Department of Nutritional Sciences, University of Arizona, Tucson, AZ, USA
Correspondence: Siew Sun Wong Oregon State University, Ballard Hall I05-E, Corvallis, OR 9733I, USA

$\mathrm{Tel}+\mathrm{I} 54 \mathrm{I} 7375855$

Fax + I 54| 7370999

Email siewsun.wong@oregonstate.edu
Abstract: The primary purpose of this review is to answer three research questions: 1) What are the most popular features of physical activity (PA) tracking and recording mobile applications (apps); 2) what features drive the app cost, peak rank, or gross sales; and 3) to what extent are evidence-based weight loss recommendations used in PA apps? Two hundred top grossing iOS health care and fitness apps were screened using a systematic review method. Fifty-five apps met the criteria as PA tracking and recording apps. Nearly half of these iOS PA apps are also available in Android. Two separate reviewers evaluated each PA app using 48 features. The top three most popular features are the use of behavioral strategies, use of the FITT Principles (frequency, intensity, time, type), and the use of the Principles for Physical Fitness. Free apps contain a mean of nine of the ten most popular features in paid apps, making them good bargains in PA promotion. Better peak rank is associated with the use of Fitness Principles, weight loss goal setting, or training videos. Ten PA apps met at least four of eight of the American College of Sports Medicine (ACSM) recommendations for weight loss and prevention of weight regain for adults. The least popular features were PA safety and workouts for special need populations. Implications for obesity treatment are discussed in relationship to individual end-users, health care providers, and app developers. Because current PA apps still lack validity and compliance to standards for PA safety and data security, medical consultation for weight loss is recommended. The quality of PA apps on the market will improve through innovative support from the government, industry, and academia. The future of PA apps is bright, especially with the potential of using emerging technologies that involve low-cost wearable sensors, global information systems, and immersive technology such as virtual worlds and virtual reality.

Keywords: Android, exercise, iOS, mHealth, self-monitoring, technology

\section{Introduction}

The primary purpose of this review is to answer three research questions by providing a narrative review on mobile applications (apps) that track and record physical activity (PA), evaluating the implications of popular features for obesity treatment among the general public. Our research questions are: 1) what are the most popular features; 2) what features drive the app cost, peak rank, or gross sales; and 3 ) to what extent are evidence-based weight loss recommendations used in PA apps? To demonstrate the importance of utilizing technology to prompt behavior change, we will first start out addressing the need for PA (eg, highlighting the obesity epidemic), traditional methods used to promote PA, emerging technological methods used to promote PA, trends in health care and fitness apps, and common features of PA tracking and recording apps used to promote PA. 


\section{Obesity prevalence and consequences of obesity \\ Obesity prevalence}

The prevalence of obesity in the US, defined as a body mass index (BMI) of $30 \mathrm{~kg} / \mathrm{m}^{2}$ or higher, did not change between 1960 and $1980,{ }^{1}$ but increased by 8.0 percentage points over the next decade (22.9\% in 1988-1994); a similar increase occurred over the following decade (30.5\% in 1999-2000), ${ }^{2}$ with a smaller change over the next time period $(33.8 \%$ in 2007-2008). ${ }^{3}$ However, more recent data demonstrates a slowing or levelling off of these trends, with $35.9 \%$ of US adults in 2009-2010 considered obese ${ }^{4}$ this prevalence estimate is not significantly different compared to the 2003-2008 estimate or more recent estimates in $2011-2012 .{ }^{5}$ Although speculative, this suggests that recent intervention efforts, such as the development and implementation of more feasible and palatable programs (eg, electronic-based programs), ${ }^{6}$ may be helping to attenuate this increased trajectory of obesity.

\section{Consequences of obesity}

Giving the relatively high proportion of obese adults in the US, reducing obesity rates is a public health priority, as obesity is associated with numerous short- and long-term health problems such as hyperlipidemia, hypertension, insulin resistance, gallbladder disease, respiratory problems, orthopedic complications, and cancer, to name a few. ${ }^{7}$

\section{Standard methods used to treat obesity}

Standard methods to treat obesity incorporate various assessment and management strategies. First, health care professionals will measure waist circumference along with BMI to assess the degree of obesity and the risk of obesity-related disease. ${ }^{8}$ These measurements are then combined with their overall health status to help determine the appropriate management strategies to employ. Depending on the individual, management and weight loss strategies may include dietary modification, PA promotion, behavior therapy (eg, selfmonitoring, stress management, problem solving, cognitive restricting, and enlistment of social support), pharmacotherapy, and/or weight loss surgery.

\section{Role of PA in treating obesity}

Although all the aforementioned strategies may play a pivotal role in treating obesity, PA is at the cornerstone of behavioral treatment for weight loss. Stated simplistically, weight loss occurs when energy expenditure is elevated above energy intake for a given time period. Regular engagement in PA favorably alters the composition of weight loss so that a greater proportion of weight loss comes from fat and less from fat-free mass loss. ${ }^{7}$ This is particularly important as PA, compared to diet alone, may help to attenuate reductions in metabolism that occur with weight loss given the effect that PA may have in maintaining or increasing fat-free mass, which is metabolically more active than fat mass. ${ }^{8}$ Another advantage of PA for weight loss is that it may help prepare the individual to sustain weight loss, as a substantive dose of PA is suggested to sustain weight loss (ie, 60-90 minutes daily of at least moderate intensity PA). ${ }^{9}$ Moreover, there is encouraging evidence to suggest that PA may influence other energy-balance behaviors, namely dietary behavior. For example, PA may indirectly affect other health behaviors including dietary intake by altering neurocognition. ${ }^{9,10}$ PA has been shown to increase a number of cognitive functions, including executive function, ${ }^{11}$ which is responsible for selfregulation and goal-oriented behavior. More specifically, PA may enhance a person's ability to monitor and adjust their intake, as well as suppress unhealthy impulsive dietary behaviors.

\section{Traditional PA interventions}

Various "traditional" strategies using a multicomponent approach have been employed to promote PA, which include using: informational approaches (eg, increase activity-related cognitive skills), point-of-decision prompts (eg, signs placed by elevators), community-wide campaigns, mass media campaigns, and environmental and policy approaches (eg, provide environmental opportunities through policy changes), and behavioral and social approaches. ${ }^{10}$ With regard to the latter, goal setting, social support, reinforcement through self-reward, problem solving, relapse prevention, and selfmonitoring are all key examples of behavioral and social approaches used to promote PA. ${ }^{10,11}$

\section{Emerging use of technology for behavior change}

Emerging technology, such as apps, may complement the aforementioned "traditional" strategies to promote PA, as they are an engaging way to involve adults by capitalizing on portability and affordability. Additional strategies, such as using technology, may be exactly what is needed to help increase the PA levels among adults, which is urgently needed as only $3.5 \%$ of US adults meet current PA guidelines ( $\geq 150$ minutes/week of moderate-to-vigorous PA) when PA is objectively measured. ${ }^{12}$ With regard to behavioral-related approaches to promote PA, self-monitoring is considered the cornerstone of behavioral treatment for weight loss. ${ }^{13}$ A large and growing body of research supports the role of self-monitoring for successful outcomes in behavioral 
weight loss interventions. ${ }^{14,15}$ In particular, using technology to self-monitor PA shows considerable promise in improving weight loss as it may help to increase self-monitoring adherence. ${ }^{15}$ For example, Wang et al recently showed that, compared to using a paper record, using technology to record PA had significant direct and indirect effects on weight loss through improved adherence to self-monitoring. ${ }^{6}$

In the past two decades, text messaging became mainstream in mobile health (mHealth) interventions. Four reviews have examined mobile phone text messaging to deliver health care interventions to improve health behaviors. Text messaging received good acceptance and induced significant favorable clinical outcomes in some studies, but methodological limitations and small sample sizes were highlighted. ${ }^{16-19}$ A systematic review by Buchholz et al identified ten PA text messaging intervention studies that have been conducted in adults. ${ }^{20}$ Interventions ranged from only sending out PA text messages, ${ }^{21-25}$ to combining text messages with educational materials, ${ }^{26,27}$ as well as providing counseling sessions delivered by health care providers and staff. ${ }^{28,29}$ Two studies combined a text messaging intervention with Internet technology. ${ }^{23,30}$ All of the studies used text messaging to provide tips. PA text messaging intervention studies showed promising results in improving PA levels, but these studies lacked specific details on the text messaging protocol, which makes it difficult for replication and implementation of other text messaging interventions.

\section{Why track PA?}

In addition to improving adherence to self-monitoring, tracking PA behavior is an effective strategy to increase PA behavior. ${ }^{31,32}$ Tracking PA levels can also positively influence other factors known to play a key role in initiating and maintaining PA. For example, Carels et al showed that individuals who self-monitor their PA tend to have fewer difficulties with exercise as well as greater exercise behavior and weight loss. ${ }^{33}$ In support of these findings, Gleeson-Kreig showed that self-monitoring PA was effective in enhancing self-efficacy, an individual's confidence in overcoming exercise-related barriers. ${ }^{34}$ Self-monitoring may also help to promote adoption of other evidenced-based PA strategies, such as goal-setting. ${ }^{35}$

In terms of setting behavior change goals to change dietary and exercise habits, studies have shown that computer-based programs are effective weight loss interventions for short-term weight loss and short-term weight loss maintenance. ${ }^{36-38}$ E-mailed ${ }^{39}$ or automated counseling ${ }^{40}$ further increase the effectiveness of computer-based interventions, although the value of online group chats is unclear. ${ }^{41}$
When compared to technology-supported interventions, non-technology supported interventions result in less weight loss $(<1.5 \mathrm{~kg}) .^{42,43}$ Nonetheless, questions still remain as to the efficacy of using the Internet for long-term weight loss maintenance, ${ }^{42,43}$ but these findings do provide further support of technology helping to facilitate behavior change.

\section{Trend in health care and fitness apps Types of health care and fitness apps developers}

There are a wide variety of app developers. These include individual app developers (professional and amateur), academic research groups, patient organizations or charities, corporate- or investment-backed app developers, pharmaceutical manufacturers, large consumer or retailing brands, health insurance groups, and national health systems. ${ }^{44}$

\section{Market and sales}

Since 2008, the mobile app economy has contributed to nearly half a million software development jobs in the US and 800,000 jobs across Europe ${ }^{45}$ Core platforms in the app economy include Google's Android (Google Inc., Mountain View, CA, USA), Apple's iOS (Apple Inc, Cupertino, CA, USA), RIM's BlackBerry (BlackBerry Ltd., Waterloo, Canada), Microsoft's Windows Phone (Microsoft Corp., Redmond, WA, USA), and Facebook's own apps (Facebook Inc., Menlo Park, CA, USA). ${ }^{45}$ Although iOS remains the most valuable platform in the global app economy, reaching US\$27 billion in 2013, Android is growing twice as fast in value and will outgrow iOS by the end of $2016 .{ }^{46}$ Do It Yourself (DIY) Health was one of 12 consumer trends in 2012. ${ }^{47}$ While the commercial sector remains the largest of the health and fitness app developers, DIY app resource is growing, especially in tailoring needs for small to mid-size businesses. Monthly service fees start at approximately US\$20 and cross platforms service fees start at nearly US $\$ 1,000 .{ }^{48}$

\section{Governmental support}

To complement the fast-growing app economy, government agencies worldwide have begun to develop expert content health and fitness apps to promote wellness and manage chronic diseases. In the US, the Office of the National Coordinator for Health Information Technology (ONC) was established in 2004 to coordinate nationwide efforts to implement and use the most advance health information technology and the electronic exchange of health information to improve health care. ${ }^{49}$ In September 2013, the US Food and Drug Administration (FDA) drafted new, yet nonbinding recommendations, to regulate mobile medical apps, starting with high-risk apps that perform complex calculations using 
patient data to aid diagnosis or therapy. ${ }^{50}$ Apps that provide users with the ability to $\log$ life events, retrieve medical content, or communicate with clinicians or health centers will not be regulated under its jurisdiction. ${ }^{50}$

The US Government Mobile App Gallery currently contains 40 apps related to health and fitness and ten related to lifestyle. ${ }^{51}$ The US Centers for Disease Control and Prevention's (CDC) Healthy Swimming app informs users about the health benefits of swimming and water safety, and Breathe2Relax informs users about the effects of stress on the body and teaches diaphragmatic breathing to manage stress. ${ }^{51}$ To date, although there is not a government agency app that tracks and/or records PA, the Center for Nutrition Policy and Promotion (CNPP) is synergistically promoting health and fitness by sharing high-quality nutrition and PA databases used in the US Department of Agriculture (USDA) "SuperTracker" with app developers via data.gov. ${ }^{52}$ The National Institutes of Health (NIH) mHealth Training Institute is offering training for behavioral and social scientists, medical professionals, and engineers to collaboratively conduct mHealth interventions and design new methodologies to conduct robust evaluation. ${ }^{53}$

In 2002-2004, The European Commission took a holistic approach by funding a telemonitoring mHealth project called MobiHealth. ${ }^{54}$ The MobiHealth consortium united 14 partners from five European countries. Partners in multiple disciplines included hospitals and medical service providers, universities, mobile network operators, mobile application service providers, and mobile infrastructure and hardware suppliers. The MobiHealth system allowed patients to be fully mobile to pursue daily life activities while undergoing health monitoring outside of the hospital. Patients wore the MobiHealth Body Area Network (BAN), a lightweight-monitoring system customized to the patients' individual health needs. In 2007, MobiHealth became a Dutch company that partners with 70 entities in more than 13 countries worldwide. Its partners include sensor manufacturers, care institutions, academic institutions, government, and companies.

\section{Media behavior of health care and fitness apps users}

As of May 2013, Pew Research Internet surveyed 2,252 American adults about smartphone ownership. It reported that $56 \%$ of American adults own a smartphone. Among them, $81 \%$ were between 25-34 years old and ownership increased with education level and income. ${ }^{55}$ In the US, fitness technology is the fastest growing sector, which rose from $8 \%$ to $33 \%$ within 1 year from 2012 to $2013 .{ }^{56}$ Fitness apps have improved considerably, allowing smartphone owners to use their phones as pedometers, accelerometers, and distance trackers, empowering consumers to assess their fitness levels, set achievable goals, track progress, and make exercise more rewarding. ${ }^{56,57}$

\section{Common features on the vendor market Star rating}

On a five-point scale, users rate an app from one to five stars. Common criteria for rating include features, interface (eg, ease of use, logic of symbols, and buttons), graphics (eg, clarity and relevance), stability (frequency of crashes or freezing, loading speed), program size, and support (eg, online documentation and contact email addresses).

\section{Cost}

Apps are either free or paid. The key differences between them include: paid apps are free of advertisements and they tend to contain more features and more extensive data storage for tracking and integration with other apps.

\section{Security}

Current PA apps on the vendor market still lack validity and compliance to standards for PA safety and data security. Free apps, in particular, may be more prone to third-party advertisement libraries that may in turn increase the risk of breaching users' personal private information such as physical location and personal contact lists. ${ }^{58}$ These issues may manifest more in the open-source Android apps market. ${ }^{59}$

\section{Sales}

Key indicators of sales include gross sales, peak rank, days in market, market stability (days in market divided by peak rank; the larger the number, the greater the stability).

\section{Hardware and software}

The most common platforms for existing PA apps are iOS and Android. Besides the processor and memory, chips are hardware components that give different functionalities to PA tracking/recording apps. Most commonly, these functionalities include: touch screen, camera, audio playback, Internet browsing, file sharing, accelerometer, gyroscope, and/or Global Positioning System (GPS). In terms of software, there are three common types of apps: native, mobile Web, and hybrid @ cross-platform. ${ }^{60}$ The native apps are programmed specifically to a given mobile platform, eg, iOS or Android. Once downloaded, a native app runs locally in the machine 
language that the platform supports. ${ }^{60}$ Mobile Web apps use Web technologies such as HTML5 and JavaScript that work across multiple platforms. No download is required and the users simply access the app over the Web. ${ }^{60}$ Hybrid apps are combinations of native and mobile Web apps. They run on the device and build on the Internet browser engine to access the device capabilities that are not accessible in mobile Web apps, such as the accelerometer, camera, and local storage. ${ }^{61}$ Most top grossing PA apps are native and hybrid apps. Automatic software updates are programmed in iOS 7.0 or later. Otherwise, users will need to manually update older app versions to keep up with operating system software compatibility. The program size represents the memory space in megabytes that is needed to install and execute the app on the device. To increase accessibility, increasing numbers of apps are providing data integration, where users can securely export encrypted personal PA data (eg, progress report, photos, comments) to online file storage websites (eg, Dropbox) or compatible apps. Real-time PA tracking often drains the smartphone battery and newer app versions are becoming more energy-saving.

\section{Common features of PA tracking/ recording apps \\ Mobile Fitness Coach}

Rabin and Bock reported that sedentary adults specifically preferred apps that automatically track PA (including energy balance), allow them to set goals, problem solve, and track progress toward PA goals for several types of PA. ${ }^{62}$ Among the top grossing PA apps, while some primarily target a specific type of PA, such as cardiorespiratory endurance, some also target cross-training. Training duration indicates the timeframe when users will meet their set goals, eg, 8 weeks for a $5 \mathrm{~km}$ run. Instructional videos are common features in muscular strength and flexibility training apps. Often, high definition video of movements is either demonstrated by a human instructor or an animated character. Some apps provide audio instruction that is transformed into a voice coach character that provides audio instructions for the entire workout session, which may include some or all of these components: warm up, interval training, cool down, safety, and verbal encouragements. Kranz et al reported that smartphones, being pervasive devices, are capable of providing expert-level feedback like a fitness coach that keeps the user's motivation longer than with conventional approaches through the automated sensing and computing capabilities. ${ }^{63}$ Training for special populations, such as pregnant mothers, is offered in some apps. ${ }^{64}$

\section{Gamification}

Gamification to motivate behavior change has shown plausible impact. For example, mobile app games helped increase fruits and vegetable consumption in an elementary school cafeteria. ${ }^{65,66}$ In “Zombies! Run!” (v2.4; Six to Start Inc., London, UK), users turn into a player who is immersed in a narrated mission and sound effects of the scene. In this case, it is the sound of zombies chasing the player as he/she runs to complete quests. The app uses GPS to convert PA into a route run on maps. The use of GPS not only requires continuous Internet connection but it also requires high-speed connectivity to achieve the best gaming experience.

\section{Data collection}

Many cardiorespiratory endurance apps, especially those for walking, running, and cycling, provide real-time, automatic PA tracking and recording. This self-monitoring feature requires continuous Internet connection. Users may also pair PA tracking and recording with Bluetooth accessories such as a heart rate monitor that synchronizes with the app data collection. Age, sex, and body composition such as height, weight, and body fat can be tracked to monitor progress. The calendar feature allows users to track and schedule workouts.

\section{Authoritative resource}

These resources refer to scientific evidence--based health and fitness guidelines and recommendations such as the FITT Principle (frequency, intensity, time, type), ${ }^{67}$ Principles of Physical Fitness (overload, progression, specificity, reversibility, individuality, and rest and recovery), and the American College of Sports Medicine (ACSM) for weight loss and prevention of weight regain for adults. ${ }^{68}$

\section{Reward}

In addition to perceptions of accomplishment from exercise (intrinsic reward), extrinsic rewards come in the form of cumulative points, badges, listing in hall of fame from local to global, price of choice in exchange with points earn, and in-kind donations to identified entities. Utilization of extrinsic factors may help to, at least initially, foster intrinsic motivation.

\section{Social networking}

This feature allows users to share personal data with people within private or public social networks. Users may also connect with coaches and athletes worldwide to exchange information to engage in joint trainings, only at different geographical locations. Users may also share real-time PA data, such as current running routes, with family and friends 
to keep them informed and/or to invite them to participate together; with regard to the latter, enlisting social support in this fashion may help to promote long-term PA compliance, as social support is a major determinant of PA maintenance and source of motivation. ${ }^{69}$ Therefore, GPS exergames (electronic, location-based games that are played outdoors with the help of GPS on mobile devices carried by players) and geosocial apps (apps that combine GPS exergames with social networking) are gaining acceptance rapidly, especially among young people, and this may be an effective way to increase PA levels. ${ }^{70}$ Web-based social networking sites, such as Facebook and Twitter are leveraging avenues. Facebook has more than 845 million users worldwide. ${ }^{71}$ These social media can facilitate direct, one-to-many communication with a large audience at little to no cost. Social media platforms are increasingly used by health care providers and practitioners to share information during training and practice. ${ }^{72}$ Additional evidence is needed on how health organizations can most effectively use this new tool for healthy behavior changes in individuals as well as for group interventions.

\section{Materials and methods}

With the purpose of identifying key features of the bestselling PA apps on the vendor market, we focused our searches on top grossing apps reports. Using the phrase and keywords of "top grossing health and fitness apps" and "activity", 2.53 million results were found. The major theme was “Top 100 grossing". To expand beyond the 100 top grossing apps, we reviewed the Top 200 grossing health and fitness apps report downloaded from the iTunes Store at $12.30 \mathrm{pm}$ Pacific Standard Time (PST) on November 15, 2013. Electronic searches on PubMed, MEDLINE, and Web of Science, were conducted until March 2014. Information from scientific journals, Consumer Reports, market reports, and articles published in English were included in the review.

\section{Review process}

The review process involved four steps. First, we compared the total number of health care and fitness mobile apps between the iTunes Store and Google Play. This resulted in 500 apps in the iTunes Store (iOS platform) and 250 in Google Play (Android platform). Since the largest number of health care and fitness apps was available in the iTunes Store, we downloaded the top 200 grossing health care and fitness apps from the iTunes Store. Our review is based on a snapshot downloaded at 12.30 pm PST on November 15, 2013. Second, we screened all 200 top grossing apps to identify native and hybrid apps that met these two criteria:
1) tracked and/or recorded PA, and 2) available in English. Apps that did not contain a function to track or record PA were excluded. For example, workout timers and instructional videos that did not have a PA tracking function were excluded. This resulted in 55 apps for the review of this paper. Third, we downloaded these apps and their descriptions and screenshots from the iTunes Store and Google Play. Using the 48 features listed in Table 1, two reviewers evaluated each app independently and reconciled all discrepancies prior to data analyses. Fourth, 4 months later (March 15, 2014), we tracked all 55 apps to evaluate changes in their features.

\section{Statistical analyses}

SPSS software for Windows (v21; IBM Corporation, Armonk, NY, USA) was used in all analyses. Descriptive statistics, Pearson correlations, and linear regression were used to describe trends and associations. Covariates were identified and stepwise multiple regressions were used to analyze the main effects between the common features of the apps with the outcome variable being either cost of the app, gross sales, or peak rank.

\section{Results \\ Overview of 55 PA tracking or recording apps}

Nearly half of the iOS apps were available in the Android platform. Breakdown of apps by type include: $47.3 \%$ cardiorespiratory endurance, $12.7 \%$ muscular strength, $1.8 \%$ flexibility and balance, and $38.2 \%$ various combinations of the PA types. Seventy-eight percent of the apps were paid (US\$0.99-9.99) and 22\% were free. Table 2 shows the characteristics of the 55 PA tracking and recording apps that were accessed on November 15, 2013. Four months later, the most noteworthy changes include: 29 (53\%) apps have a newer version for the main reasons of debugging and increasing compatibility to an updated version of the operating system; 13 (24\%) apps added remarkable new features; and four (7\%) apps had a price drop.

\section{What are the most popular features?}

The ten most popular features of the 55 reviewed apps are summarized in Table 3. The top three features are: Rank \#1) use of behavioral strategies for weight loss; Rank \#2) use of FITT Principles; and Rank \#3) use of the Principles of Physical Fitness. Both free and paid apps used at least one of the behavioral strategies for weight loss (particularly self-monitoring and social support) and the FITT Principles. At least $75 \%$ of apps containing these features had an 
Table I Identified features from 55 evaluated PA tracking and recording apps

I. User star rating (lowest rating $=\mid$ star, highest rating $=5$ stars)

2. Cost of the mobile app in US dollars

3. Gross sales in US dollars

4. Peak rank reached during the lifetime of the app

5. Number of days on the market

6. Market stability (days in market divided by peak rank. The greater the number, the stronger the market stability)

7. Version of mobile application being reviewed

8. Software program size (megabytes)

9. Is the app available in Android platform?

10. Does the app require Internet connection to track/record movements in real-time?

II. Does the app automatically track/record movements without manual entries?

12. Does the app require Global Positioning System (GPS) to track/ record movements?

13. Does the app require an additional gadget to measure the movements?

14. Is a real-time, personalized voice coach available during training?

15. Does the app contain instruction videos? The video may feature a real-person trainer or a graphic animation of a trainer

16. Does the app allow the users to create their own movements? For example, users record and upload new personalized workout videos or instructions

17. Is there instruction on warm up and cool down during training?

18. Is safety mentioned?

19. Does the training have a special module for users with special condition such as pregnant mothers?

20. Duration of training course to achieve goal (eg, to complete a $5 \mathrm{~km}$ run)

21 . Is the workout gamified? (Players become immersed in a game while engaging in PA movements)

22. Is reward used? (eg, points, gifts, donations etc)

23. Is this app merely a calendar for users to track their workout schedules?

24. Is the data exportable to other sites or apps?

25. Number of other apps in which personal data can be accessed/shared

26. Body composition: is age recorded?

27. Body composition: is sex recorded?

28. Body composition: is weight recorded?

29. Body composition: is height recorded?

30. FITT Principle: type of PA measured (cardiorespiratory, strength, flexibility, and/or various combinations of them)

3I. FITT: type of PA measured is cardiorespiratory endurance

32. FITT: type of PA measured is muscular strength and endurance

33. FITT: type of PA measured is flexibility

34. FITT: total number of PA types measured

35. FITT: is PA frequency measured?

36. FITT: is PA intensity measured?

37. FITT: is PA time (duration) measured?

38. Principles of Physical Fitness: did training use any of the six principles: overload, progression, specificity, reversibility, individuality, and rest and recovery?

39. ACSM recommendation \#I: Does the app calculate BMI?

40. ACSM recommendation \#2: Does the app allow users to set weight loss goal?

41. ACSM recommendation \#3: Does the app allow users to track their progress in weight loss? (We included tracking of progress in PA movements because this also supports weight loss)

(Continued)
Table I (Continued)

42. ACSM recommendation \#4: Does the app use behavioral strategies?

43. ACSM recommendation \#5: Does the app also allow users to set dietary goals?

44. ACSM recommendation \#6: Does the app build in different levels for users to advance? (progress in intensity and time/duration)

45. ACSM recommendation \#7: Does the app add resistance training to its primary training purpose?

46. ACSM recommendation \#8: Does the app recommend pharmacotherapy?

47. Total number of ACSM recommendations met

48. Percentage of ACSM recommendations met

Abbreviations: ACSM, American College of Sports Medicine; app, mobile application; BMI, body mass index; FITT, frequency, intensity, time, type; PA, physical activity.

Android version. Rank \#4 is progression. About two-thirds of these apps allow users to progress to advancing levels during the course of training to accommodate different level needs. Ranks \#5 and \#6 are maintenance and data interoperability. Slightly more than half of the apps allow users to track their progress or amount of PA with time, some for up to 1 year. Data interoperability was higher among these apps, which allow users to export or share their personal PA-related data with other platforms such as another app, online shared drive, Microsoft Excel, and emails. Rank \#7 is tracking and recording of body composition, particularly self-reported height and weight. Tracking or recording of waist circumference was not observed among the 55 apps reviewed. Rank \#8 is real-time tracking, which requires Internet connectivity, especially for apps that integrate GPS in tracking and recording PA. Rank \#9 is voice coach and Rank\# 10 is reward system. Both features were key motivators to users during training and upon reaching their goals.

By comparing the number of top ten features found in free and paid apps, there was no indication of paid apps being better than free apps. In fact, nine of the ten features were more common in the free apps. Maintenance was the only feature that was more common in paid apps, most likely due to increased service fees for secure data storage of personal confidential data.

Table 2 Characteristics of 55 evaluated PA tracking and recording apps

\begin{tabular}{ll}
\hline $\begin{array}{l}\text { Average number of PA types per apP } \\
\text { (cardiorespiratory, muscular strength, and/or flexibility) }\end{array}$ & I.56 \\
Average cost & US $\$ 3.39 \pm 1.62$ \\
Range of training duration & $4-16$ weeks \\
Average number of days on market & $191 \pm 209$ days \\
Average program size & $50.15 \pm 75 \mathrm{MB}$ \\
Number of apps with both iOS and Android platforms & $27(49 \%)$ \\
\hline
\end{tabular}

Abbreviations: app, mobile application; PA, physical activity. 
Table 3 Top ten most popular features of PA tracking and recording apps

\begin{tabular}{lllll}
\hline $\begin{array}{l}\text { Top ten features ranked } \\
\text { in descending order }\end{array}$ & $\begin{array}{l}\text { Total counts } \\
\text { (\% apps) }\end{array}$ & $\begin{array}{l}\text { \% free apps with } \\
\text { this feature }\end{array}$ & $\begin{array}{l}\text { \% paid apps with } \\
\text { this feature }\end{array}$ & $\begin{array}{l}\text { \% apps that also have } \\
\text { an Android platform }\end{array}$ \\
\hline I. Use behavioral strategies & $55(100 \%)$ & $100 \%$ & $100 \%$ & $100 \%$ \\
2. Use FITT Principles & $49-55(89 \%-100 \%)$ & $100 \%$ & $100 \%$ & $89 \%-96 \%$ \\
3. Use Principles of Physical Fitness & $44(80 \%)$ & $92 \%$ & $81 \%$ & $75 \%$ \\
4. Progression & $37(67 \%)$ & $92 \%$ & $53 \%$ & $64 \%$ \\
5. Maintenance & $32(58 \%)$ & $42 \%$ & $63 \%$ & $67 \%$ \\
6. Data interoperability & $32(58 \%)$ & $58 \%$ & $58 \%$ & $60 \%$ \\
7. Track and record body composition & $22-29(40 \%-53 \%)$ & $83 \%$ & $60 \%$ & $46 \%$ \\
8. Real-time tracking & $28(51 \%)$ & $67 \%$ & $44 \%$ & $50 \%$ \\
9. Voice coach & $27(49 \%)$ & $58 \%$ & $47 \%$ & $53 \%$ \\
10. Use reward system & $22(40 \%)$ & $58 \%$ & $35 \%$ & $29 \%$ \\
\hline
\end{tabular}

Abbreviations: app, mobile application; FITT, frequency, intensity, time, type; PA, physical activity.

\section{What features drive the app cost, peak rank, or gross sales?}

No single feature we examined drove the app cost $(P>0.1)$. As for peak rank (the smaller the number, the higher the rank), negative associations were found between three features: 1) use of at least one of five Principles of Physical Fitness $(r=-0.39, P<0.01) ; 2$ ) use of ACSM recommendation \#2, ie, set weight loss goal $(r=-0.31, P<0.05)$; and 3$)$ the use of training videos $(r=-0.281, P<0.05)$. These results suggest that apps would have a better peak rank if they integrate at least one of five Principles of Physical Fitness, weight loss goal setting, and/or training videos. For gross sales (the smaller the number, the higher the rank), overall ranking of gross sales increased with more ACSM recommendations met ( $r=-0.317, P<0.05$ ). Apps that were mainly designed for planning workout ranked poorer in peak rank $(r=0.33$, $P<0.05)$ and gross sales $(r=0.384, P<0.05)$. Multiple linear regression tests did not show any multi-feature influence on cost, peak rank, and total gross rank.

\section{To what extent are evidence-based weight loss recommendations used in PA apps?}

We evaluated all 55 apps in the extent of meeting the 2009 ACSM recommendations of weight loss and prevention of weight regain among adults. ${ }^{59}$ On average, $44 \%$ of ACSM recommendations were met. Table 4 shows a breakdown of the number and percentage of apps that met individual ACSM recommendations. The top three ACSM recommendations met were \#4 (behavioral strategies for weight loss), \#6 (progression), and \#2 (set weight loss goal). The calculation of BMI was only available in six apps and dietary goal setting was only available in eight apps.

We further evaluated apps that met more than half of the ACSM recommendations. Table 5 shows descriptions of ten apps that met this criterion. Half of them had a peak rank of 1 at some point among all health care and fitness apps available in the iTunes Store.

\section{Discussion}

For the purpose of relating the findings from this study to the implications for obesity treatment, the following discussion of the results, current challenges, and potential solutions will be presented in terms of their relationship to three major groups of audience: 1) individual end-users, 2) health care providers, and 3) app developers.

\section{Individual end-users}

The Consumer Electronics Association (CEA) reported that one-third of US consumers planned to purchase fitness technologies in $2013 .{ }^{73}$ Perhaps because the rate of adult obesity has not changed significantly between 2003-2004 (35.9\%) and 2011-2012 (34.9\%), 5 the popularity of health care and fitness apps that target individuals wishing to lose weight increased. Our results show that free apps actually

Table 4 The extent of PA tracking/recording apps using ACSM recommendations for appropriate $\mathrm{PA}$ intervention strategies for weight loss and prevention of weight regain for adults

\begin{tabular}{llll}
\hline ACSM recommendations (2009) & $\mathbf{n}$ & $\%$ \\
\hline Rec \#I: Does the app calculate BMI? & 6 & II \\
Rec \#2: Does the app allow users to set weight loss goals? & 22 & 40 \\
Rec \#3: Does the app allow users to track their progress & 32 & 58 \\
in weight loss? & & \\
Rec \#4: Does the app use behavioral strategies? & 55 & 100 \\
Rec \#5: Does the app also allow users to set dietary goals? & 8 & 14 \\
Rec \#6: Does the app build in different levels for users & 37 & 67 \\
to advance? & & \\
Rec \#7: Does the app add resistance training & II & 20 \\
to its primary training purpose? & & \\
Rec \#8: Does the app recommend pharmacotherapy? & I & 2 \\
\hline
\end{tabular}

Abbreviations: ACSM, American College of Sports Medicine; app, mobile application; BMI, body mass index; PA, physical activity; Rec, recommendation. 
Table 5 PA tracking and recording apps that met more than half of the 2009 ACSM recommendations for weight loss and prevention of weight regain among adults

\begin{tabular}{|c|c|c|c|c|c|c|}
\hline App name & $\%$ ACSM rec met & Gross rank & Peak rank & Developer & Version & Cost (US\$) \\
\hline All-in Fitness: 1000 Exercises, Workouts, Calorie & $88 \%$ & 18 & I & Sport.com & 1.4 & $\$ 2.99$ \\
\hline \multicolumn{7}{|l|}{ Counter, BMI-calculator and Social Network } \\
\hline Full Fitness: Exercise Workout Trainer & $75 \%$ & 51 & 1 & Health Xperts Inc. & 2.2 & $\$ 1.99$ \\
\hline Running for Weight Loss PRO: training plan, & $75 \%$ & 6 & I & Grinasys Corp. & I.2.1 & $\$ 3.99$ \\
\hline \multicolumn{7}{|l|}{ GPS, how-to-lose-weight tips by Red Rock Apps } \\
\hline JEFIT PRO Workout - Fitness and Exercise & $75 \%$ & 108 & 16 & Jefit Inc. & 7.3 & $\$ 4.99$ \\
\hline \multicolumn{7}{|l|}{ Tracking System } \\
\hline Run with Map My Run - GPS Running, Jog, & $63 \%$ & 34 & 2 & MapMyFitness Inc. & 4.6.1I & Free \\
\hline \multicolumn{7}{|l|}{ Walk, Workout Tracking and Calorie Counter } \\
\hline Map My Fitness & $63 \%$ & 81 & 33 & MapMyFitness Inc. & 5.0 .4 & Free \\
\hline RunKeeper - GPS Track Running Walking Cycling & $63 \%$ & 9 & 2 & FitnessKeeper, Inc. & 4.1 & Free \\
\hline Fitness Buddy: 1700+ Exercise Workout Journal & $63 \%$ & 8 & 1 & Azumio Inc. & 3.1 & $\$ 1.99$ \\
\hline Map My Fitness+ & $63 \%$ & 74 & 12 & MapMyFitness Inc. & 5.0 .4 & $\$ 2.99$ \\
\hline Runtastic PRO GPS Running, Walking and Fitness & $63 \%$ & 13 & 1 & Runtastic GmbH & 3.1 & $\$ 4.99$ \\
\hline Tracker & & & & & & \\
\hline
\end{tabular}

Abbreviations: ACSM, American College of Sports Medicine; app, mobile application; BMI, body mass index; GPS, Global Positioning System; PA, physical activity; rec, recommendations.

contain more top ten features reviewed than paid apps. We did not find any association between app cost and the number of features. This means, with an average cost of US\$2.65 per PA app, free PA apps are actually good bargains for end-users. Most of these iOS PA apps are also available on the Android platform, which is projected to become more popular, thus expanding the reach to noniPhone users.

For users seeking weight loss through the use of PA apps, we recommend completing the Physical Activity Readiness Questionnaire (PAR-Q) as the first step. After discussing the PAR-Q response with a doctor, users may choose PA apps that meet as many ACSM recommendations and evidencebased PA guidelines as possible. For users with medical conditions and high-risk for PA-related adverse effects or injuries, we recommend seeking advice from medical doctors before following any app's exercise routine. Because current commercial PA apps (especially non-medical apps) still lack US FDA regulations to meet safety and confidentiality requirements, we do not recommend using these PA apps alone for weight loss without medical advice and monitoring. Furthermore, the accuracy of data outputs such as total step counts and calories burned has not been empirically tested for validity and reliability. ${ }^{74}$ Therefore, end-user patients are encouraged to interpret these outputs with caution. The accuracy of data outputs may be improved by using validated wearable sensors specifically designed for professional use. However, users may risk losing expensive wearable sensors, as these devices are typically clipped on to clothing or worn as a wrist or an arm band.

\section{Health care providers}

Our results show a limited number of apps meeting ACSM recommendations for weight loss and prevention of weight regain for adults. Only one of 55 PA apps considered the users' medical condition by tracking the use of supplements, and only one app provides exercise routines for pregnant mothers.

Mobile apps can provide a fast and efficient way for doctors and patients to stay in touch and exchange information. ${ }^{60} \mathrm{~A}$ few research and innovative mHealth apps allow users to measure vital signs and biological markers either using the camera on the smartphone or external Bluetooth devices, including a heart rate monitor, blood pressure monitor, digital weighing scale, glucometer, and a device that measures skin vitamin A levels. Patients will have more power to selfmanage their conditions (eg, through reminders to check their blood glucose after exercise), which may reduce in-person appointments while maintaining quality diagnosis (symptoms checkers), general health advising, and treatment.

A survey among 131 junior doctors in the UK found that junior doctors would like future apps to focus on providing clinical information at the point of care and decision support for prescriptions and treatments. ${ }^{60}$ Today, there is limited information about PA safety in the top grossing PA apps. Medical content accuracy and the security of these apps still require regulation. There is also limited justifiability as health benefits are mostly personal opinion from coaches and anecdotal testimonies from other users. As a result, physicians still hesitate to formally recommend PA apps to patients. 
In future, the inclusion of ACSM recommendations and other PA guidelines in specialized medical PA apps for physicians as end-users would be very useful to help them make recommendations to their patients. Support for health care providers may include professional guidance on app quality documented with credible scientific evidence, trainings for app use in practice, and ethics and confidence in the security of personal health information. ${ }^{44}$

In the evolution of health care stakeholders, patients are increasingly involved in health care decision making and choices. ${ }^{44}$ Physicians may interact with their patients via the app social network not only as a health care provider but ideally, also as an end-user of the creditable PA app. Future apps could link a patient's screening results, such as the PAR-Q, vital signs, and daily PA records to their electronic health records. Scaling up the MobiHealth system globally will truly allow patients and physicians to become mobile worldwide while providing more confidence in access to an individual's electronic health record anywhere at their fingertips.

\section{App developers}

The results of our review show that $100 \%$ of the PA tracking and recording apps used at least one behavior strategy for weight loss to promote PA. However, when we evaluated them against evidence-based weight loss recommendations, the result was less encouraging. Only ten (18\%) of 55 apps met more than half of ACSM recommendations for weight loss and prevention of weight regain. ${ }^{68}$ It is important for individuals seeking weight loss treatment and prevention of unhealthy weight gain to follow a program that meets the ACSM guidelines not only because of its effectiveness, but also for safety reasons. We highly recommend app developers to apply and integrate more ACSM recommendations into PA apps in future, including an automated PAR-Q to assess the user's readiness to engage in PA.

Effective and timely feedback is an important component in motivating users to keep tracking their PA and engaging in weight loss. Voice coach, as one of the ten most popular features, is a good example of real-time feedback during exercise. However, due to standard programming, personalized feedback in response to actual health condition and progress made by users is either none or very limited. Applying artificial intelligence (AI) in smartphones is a promising field in which to experiment. It would be extremely helpful if an AI-integrated smartphone could measure the fidelity of human performance through processes and creditable reasoning to provide personal feedback given what it knows. This may even expand from the individual level to family level, and to community level to support patients who are undergoing obesity treatments. This may be achieved by increased interoperability of electronic health records through vigorous and meaningful collaboration between the health care system and the app industry.

\section{Market penetration}

To date, only $18 \%$ of the $\geq 65$ years population uses a smartphone, compared to $55 \%$ of those between 45 and 54 years old. This older population comprises individuals who typically face multiple chronic diseases with the greatest medical needs. ${ }^{44}$ Furthermore, there is a significant increase in obesity among women aged 60 years and older in the US. ${ }^{5}$ Therefore, there is great need for smartphone penetration to occur among the aging population.

\section{Platforms and cost}

It will be advantageous for app developers to become familiar in different programming environments to be competitive in penetrating the health care and fitness sector. ${ }^{60}$ Native apps, although the best by far in terms of performance, especially in leveraging the devices' hardware so seamlessly, are still more expensive than hybrid apps and mobile Web apps. Furthermore, apps programmed for operating systems older than iOS7 do not automatically update, thus causing problems in older versions.

App developers may increase hybrid apps, ie, apps that embed HTML5 apps inside a native container that provides access to native platform features and their built-in components, such as the camera, address book, geo-location, or any other features native to the device ${ }^{64}$ to use the devices' hardware seamlessly and to increase consumer reach.

Alternatively, depending on needs, resources, and prioritization, a new app can be tested as a Web app before it is developed into a native app or a hybrid app. Web apps provide automatic updates, and in general, provide good value to consumers. However, Web apps are slower than native and hybrid apps, and tracking stops when the user is offline. Another downside of Web apps is the partial access to the smartphone hardware that limits its capacity to track and record PA conveniently. Nonetheless, it is cheaper to develop.

\section{Compliance}

US FDA regulations for mHealth apps are being shaped in response to the fast growing numbers of mHealth apps. The US FDA has proposed the regulation of medical devices that are apps intended to perform calculations, enhancements, and interpretations of entered or captured patient data. ${ }^{50}$ The level 
of risk will be determined. For example, if an app performs complex calculations using patient data to aid diagnosis or therapy, it is classified as a high-risk medical device. ${ }^{60}$ The Health on The Net Foundation is another entity that provides a certification process to ensure that apps do not pose potential harm to their users or have significant security and privacy vulnerabilities. ${ }^{75}$ These certification processes will standardize the terminologies used in apps and to ensure apps are designed to support, not replace, the relationship that exists between a patient and their existing physician. Immediate attention should be given to medical apps and sensors that are linked to medical devices and medical services delivered through apps. This is because these apps may face regulatory barriers or uncertainties even where the balance of benefit versus risk favors freedom to innovate. ${ }^{76}$

\section{Support needed to improve the quality and sustainability of mHealth apps that measure PA}

In the long run, innovative and sustainable support from the government, industry, and academia are needed to improve the quality of future mHealth apps. The following are key components in this process.

\section{Review and certification}

Establishing an unbiased review and certification process is a key step in helping mHealth apps achieve their potential in improving health and wellness. ${ }^{77}$ App review organizations such as the Health On the Net Foundation and Underwriters Laboratories may expand to review more apps through timely and rigorous yet meaningful certification processes. It is important to ensure that mHealth apps do not pose potential harm to their users or have significant security and privacy vulnerabilities. ${ }^{77}$ The $\mathrm{ONC}^{49}$ could help support the development of mHealth app guidelines and eventually commission app certification entities. ${ }^{77}$ This will provide viable means of improving the quality of mHealth apps via free or low-cost certification

\section{Partnership between governmental sector, private sector, and non-profit organizations}

None of the 200 top grossing health care and fitness apps we reviewed were developed by the government or in partnership with the government. It is time for the government to partner with the app industry. Incentives should be given to app developers who provide precise medical treatment recommendations and diagnostic information to develop apps for patients to promote more collaboration between the US FDA and the app industry.
Hospitals may identify potential quality app developers through $\mathrm{KLAS}^{\circledR}$ Research unbiased reports. KLAS conducts $>1,900$ health care provider interviews per month and works with $>4,500$ hospitals and $>3,000$ doctors' offices and clinics. It publishes approximately 40 performance and perception reports per year. Its mission is to improve health care technology delivery by measuring vendor performance for the company's provider partners. ${ }^{78}$

\section{Data integration and interoperability}

$\mathrm{ONC}$ is developing guidelines for electronic health records. This digital patient's record typically includes patient demographics, progress notes, problems, medications, vital signs, past medical history, immunizations, lab data, and radiology reports. Adding the PA records, including summaries, to this digital collection of patient health information at any care delivery setting would bring forth better informed patient care and medical decision making. To facilitate data integration, ONC could support the development of mHealth data standards ${ }^{77}$ along with incentives to app developers who are willing to share data upon users' consensus. Apps can also link to more apps or features that support the entire continuum of health care needs in a "patient journey" that consists of overall wellness, diagnosis, health care provider visits, further information, prescription filing, and compliance. ${ }^{44}$

\section{Institutional support}

Academic institutions play an important role in research and innovation. Increasing partnership with the industry is seen. To keep up with the momentum in the industry, institutional support, such as approval from Institutional Review Boards (IRB), needs to speed up with a timely review process when research involves human subjects. This act is critical in allowing researchers to catch up and hopefully, align with the rapid advancement in mHealth technology. The synergies from US FDA regulatory attention and timely IRB approval for largescale randomized control trials will accelerate meaningful and effective use of mHealth apps in health care.

\section{Strengths and limitations of this review}

The strengths of this review include: 1) 48 features of PA apps were reviewed and evaluated by two reviewers separately; 2) apps were re-evaluated 4 months later to examine changes; and 3) all PA apps were evaluated based on scientific evidence-based PA guidelines such as the ACSM recommendations and the Principles of Physical Fitness. Nonetheless, limitations in this review exist: 1) among 40,000+ health care and fitness apps, we only reviewed the top 200 grossing 
health care and fitness apps in the interest of identifying the most popular features of best-selling PA apps on the vendor market. In future, a more comprehensive review of all PA apps, including those customized in research interventions and their theoretical framework, is needed to better inform researchers, as well as consumers; 2) we did not evaluate the numbers of downloads, and assumed that the top 200 grossing apps are among the top tiers of downloads; and 3 ) in the context of the type of end-users for the top grossing PA apps reviewed, our scope of target audience was limited to the general public, which did not include patients in homecare, trauma care, and ambulatory monitoring.

\section{Future direction}

How can we utilize the positional advantage of emerging technology to treat obesity? First, the use of an innovative, safe, low-cost yet sensitive wearable sensor will enable large-scale community-based research and interventions to prevent and treat obesity. Disposable and water-resistant wearable sensors like a Band-Aid worn on the chest to track PA and its related parameters such as heart rate, body temperature, electrocardiogram, location, and time are being developed (Chiang, Oregon State University, personal communication, August, 2013). In the future, these wearable sensors-on-a-chip have the potential to cost below US $\$ 1.00$ each and have battery life that will last for more than one week (unpublished work).

In the USDA NIFA funded (2011-68001-30020) "Generating Rural Options for Weight (GROW) Healthy Kids \& Communities" project, ${ }^{79}$ John and Gunter's multistate team is using community-engaged research and participatory interventions to map local food and PA resources in rural community locations in Oregon and partnering states (Washington, Idaho, Nevada, Colorado, New Mexico) with a goal of preventing obesity among rural populations. Community members, using camera-enabled GPS units, identify local sites that support healthy eating and PA. The resulting maps and GIS (geographic information system) information allow researchers, residents, and visitors to visualize the distribution of PA resources and the spatial relationship of the resources, both indoor and outdoor sites, to where people live, work, and play. Crowdsourcing may feasibly expand this innovation globally in mapping access to safe and vibrant PA indoor and outdoor resources around the world. ${ }^{79}$

Immersive technology, such as virtual world and virtual reality, is a tremendous medium to promote PA. Studies have shown that adults who exercised in the physical world and whose avatars also exercised in the virtual world had more significant weight loss and/or weight loss maintenance than those who did not have an avatar that exercised in the virtual world. ${ }^{80-82}$ Using virtual reality, players who saw themselves as an avatar getting thinner as they walked but getting fatter as they stopped walking significantly increased their PA within the next 24 hours after exposure. ${ }^{83}$ Facebook has recently purchased Oculus, virtual reality goggles that fully immerse players in a motion game. The synergies from social networking and immersive technology will accelerate PA promotion in fun and engaging ways. At the same time, a more diverse audience may also be reached including caregivers, veterans, and persons with developmental disabilities.

In the meantime, there needs to be a mechanism to help researchers find apps that meet their research needs and improve the speed of research progression. ${ }^{59}$ For example, access to a centralized archive of research-oriented and fieldtested PA apps, eg, managed by eXtension Community of Practice, may create a synergy that improves the quality (eg, content, usability, reliability, and validity) and safety (ie, privacy, confidentiality, and physical safety when engaging in the PA) of PA apps through multi-sector collaborations. For health care providers, this pool of PA apps may further enhance point of care, particularly in patient education, disease self-management, and remote monitoring of patients. ${ }^{84}$

\section{Conclusion}

The top three most popular features of top grossing PA tracking and recording apps are the use of behavioral strategies for weight loss, use of FITT Principles, and the Principles of Physical Fitness. Peak rank and gross sales rank improved with greater use of evidence-based PA guidelines such as the ACSM recommendations for weight loss and prevention of weight regain. As personal interest in weight loss increases, there is also a greater need to improve the quality of PA apps to ensure safety and personal data confidentiality to maximize benefits over risks. The future direction of using emerging technology to promote PA is bright. Partnerships between government, industry, and academia are needed to advance the mHealth movement.

\section{Disclosure}

The authors report no conflicts of interest in this work.

\section{References}

1. Flegal KM, Carroll MD, Kuczmarski RJ, Johnson CL. Overweight and obesity in the United States: prevalence and trends, 1960-1994. Int J Obes Relat Metab Disord. 1998;22(1):39-47.

2. Flegal KM, Carroll MD, Ogden CL, Johnson CL. Prevalence and trends in obesity among US adults, 1999-2000. JAMA. 2002;288(14): $1723-1727$. 
3. Flegal KM, Carroll MD, Ogden CL, Curtin LR. Prevalence and trends in obesity among US adults, 1999-2008. JAMA. 2010;303(3): 235-241.

4. Flegal KM, Carroll MD, Kit BK, Ogden CL. Prevalence of obesity and trends in the distribution of body mass index among US adults, 1999-2010. JAMA. 2012;307(5):491-497.

5. Ogden CL, Carroll MD, Kit BK, Flegal KM. Prevalence of childhood and adult obesity in the United States, 2011-2012. JAMA. 2014;311(8): 806-814.

6. Wang J, Sereika SM, Chasens ER, Ewing LJ, Matthews JT, Burke LE. Effect of adherence to self-monitoring of diet and physical activity on weight loss in a technology-supported behavioral intervention. Patient Prefer Adherence. 2012;6:221-226.

7. Must A, Spadano J, Coakley EH, Field AE, Colditz G, Dietz WH. The disease burden associated with overweight and obesity. JAMA 1999;282(16):1523-1529.

8. Cerhan JR, Moore SC, Jacobs EJ, et al. A pooled analysis of waist circumference and mortality in 650,000 adults. Mayo Clin Proc. 2014;89(3):335-345.

9. Loprinzi PD, Smit E, Mahoney S. Physical activity and dietary behavior in US adults and their combined influence on health. Mayo Clin Proc. 2014;89(2):190-198.

10. Kahn EB, Ramsey LT, Brownson RC, et al. The effectiveness of interventions to increase physical activity. A systematic review. Am J Prev Med. 2002;22(Suppl 4):73-107.

11. Heath GW, Parra DC, Sarmiento OL, et al. Evidence-based intervention in physical activity: lessons from around the world. Lancet. 2012;380(9838):272-281.

12. Troiano RP, Berrigan D, Dodd KW, Masse LC, Tilert T, McDowell M. Physical activity in the United States measured by accelerometer. Med Sci Sports Exerc. 2008;40(1):181-188.

13. Wing R. Behavioral approaches to the treatment of obesity. In: Bray GA, Bourchard C, James W, editors. Handbook of Obesity: Clinical Applications. 2nd ed. New York, NY: Marcel Dekker; 2004: 147-167.

14. Boutelle KN, Kirschenbaum DS. Further support for consistent selfmonitoring as a vital component of successful weight control. Obes Res. 1998;6(3):219-224.

15. Burke LE, Wang J, Sevick MA. Self-monitoring in weight loss: a systematic review of the literature. J Am Diet Assoc. 2011;111(1):92-102.

16. Cole-Lewis H, Kershaw T. Text messaging as a tool for behavior change in disease prevention and management. Epidemiol Rev. 2010;32(1): 56-69.

17. Heron KE, Smyth JM. Ecological momentary interventions: incorporating mobile technology into psychosocial and health behaviour treatments. Br J Health Psychol. 2010;15(Pt 1):1-39.

18. Krishna S, Boren SA, Balas EA. Healthcare via cell phones: a systematic review. Telemed J E Health. 2009;15(3):231-240.

19. Wei J, Hollin I, Kachnowski S. A review of the use of mobile phone text messaging in clinical and healthy behaviour interventions. J Telemed Telecare. 2011;17(1):41-48.

20. Buchholz SW, Wilbur J, Ingram D, Fogg L. Physical activity text messaging interventions in adults: a systematic review. Worldviews Evid Based Nurs. 2013;10(3):163-173.

21. Fukuoka Y, Vittinghoff E, Jong SS, Haskell W. Innovation to motivation - pilot study of a mobile phone intervention to increase physical activity among sedentary women. Prev Med. 2010;51(3-4): 287-289.

22. Haapala I, Barengo NC, Biggs S, Surakka L, Manninen P. Weight loss by mobile phone: a 1-year effectiveness study. Public Health Nutr. 2009;12(12):2382-2391.

23. Kim HS, Jeong HS. A nurse short message service by cellular phone in type-2 diabetic patients for six months. J Clin Nurs. 2007;16(6): 1082-1087.

24. Prestwich A, Perugini M, Hurling R. Can the effects of implementation intentions on exercise be enhanced using text messages? Psychol Health. 2009;24(6):677-687.
25. Prestwich A, Perugini M, Hurling R. Can implementation intentions and text messages promote brisk walking? A randomized trial. Health Psychol. 2010;29(1):40-49.

26. Cheung PP, Chow BC, Parfitt G. Using environmental stimuli in physical activity intervention for school teachers: a pilot study. Int Electron J Health Educ. 2008;11:47-56.

27. Lee CY, Lee H, Jeon KM, Hong YM, Park SH. Self-management program for obesity control among middle-aged women in Korea: a pilot study. Jpn J Nurs Sci. 2011;8(1):66-75.

28. Fjeldsoe BS, Miller YD, Marshall AL. MobileMums: a randomized controlled trial of an SMS-based physical activity intervention. Ann Behav Med. 2010;39(2):101-111.

29. Rossi MC, Vespasiani G, Perozzi C, et al. An interactive diary for diet management (DAI): a new telemedicine system able to promote body weight reduction, nutritional education, and consumption of fresh local produce. Diabetes Technol Ther. 2010;12(8):641-647.

30. Hurling R, Catt M, Boni MD, et al. Using internet and mobile phone technology to deliver an automated physical activity program: randomized controlled trial. J Med Internet Res. 2007;9(2):e7.

31. Kirwan M, Duncan MJ, Vandelanotte C, Mummery WK. Using smartphone technology to monitor physical activity in the 10,000 Steps program: a matched case-control trial. J Med Internet Res. 2012;14(2):e55.

32. Normand MP. Increasing physical activity through self-monitoring, goal setting, and feedback. Behav Interv. 2008;23(4):227-236.

33. Carels RA, Darby LA, Rydin S, Douglass OM, Cacciapaglia HM, O'Brien WH. The relationship between self-monitoring, outcome expectancies, difficulties with eating and exercise, and physical activity and weight loss treatment outcomes. Ann Behav Med. 2005;30(3): $182-190$.

34. Gleeson-Kreig JM. Self-monitoring of physical activity: effects on self-efficacy and behavior in people with type 2 diabetes. Diabetes Educ. 2006;32(1):69-77.

35. Schoffman DE, Turner-McGrievy G, Jones SJ, Wilcox S. Mobile apps for pediatric obesity prevention and treatment, healthy eating, and physical activity promotion: just fun and games? Transl Behav Med. 2013;3(3):320-325.

36. Baulch J, Chester A, Brennan L. Treatment alternatives for overweight and obesity: the role of online interventions. Behav Change. 2008;25(1):1-14.

37. Neve M, Morgan PJ, Jones PR, Collins CE. Effectiveness of web-based interventions in achieving weight loss and weight loss maintenance in overweight and obese adults: a systematic review with meta-analysis. Obes Rev. 2010;11(4):306-321.

38. Wieland LS, Falzon L, Sciamanna CN, et al. Interactive computer-based interventions for weight loss or weight maintenance in overweight or obese people. Cochrane Database Syst Rev. 2012;8:CD007675.

39. Steinberg DM, Tate DF, Bennett GG, Ennett S, Samuel-Hodge C, Ward DS. The efficacy of a daily self-weighing weight loss intervention using smart scales and e-mail. Obesity (Silver Spring). 2013;21(9): 1789-1797.

40. Tate DF, Jackvony EH, Wing RR. A randomized trial comparing human e-mail counseling, computer-automated tailored counseling, and no counseling in an internet weight loss program. Arch Intern Med. 2006;166(15):1620-1625.

41. Webber KH, Tate DF, Michael Bowling J. A randomized comparison of two motivationally enhanced internet behavioral weight loss programs. Behav Res Ther. 2008;46(9):1090-1095.

42. Neville LM, Milat AJ, O'Hara B. Computer-tailored weight reduction interventions targeting adults: a narrative systematic review. Health Promot J Austr. 2009;20(1):48-57.

43. Weinstein PK. A review of weight loss programs delivered via the internet. J Cardiovasc Nurs. 2006;21(4):251-258; quiz 259-260.

44. Aitken M. Patient apps for improved healthcare: from novelty to mainstream. Parsippany, NJ: IMS Institute for Healthcare Informatics; 2013. Available from: http:/www.imshealth.com/deployedfiles/imshealth/ Global/Content/Corporate/IMS\%20Health\%20Institute/Reports/ Patient_Apps/IIHI_Patient_Apps_Report.pdf. Accessed June 4, 2014. 
45. Mandel M. Where the Jobs are: The App Economy. TechNet: South Mountain Economics, LLC; 2012.

46. App economy forecasts 2013-2016: developer population, platforms, and revenue models sizing and forecasts 2013-2016 [webpage on the Internet]. Plum Consulting and Vision Mobile; 2013. Available from: http://www.visionmobile.com/product/app-economy-forecasts-20132016/. Accessed June 4, 2014.

47. 12 crucial consumer trends for 2012; 2011. Available from: http:// trendwatching.com/trends/12trends2012/. Accessed March 23, 2014.

48. Clancy H. 14 DIY mobile app development resources for small businesses. ZDNet; 2012. Available from: http://www.zdnet.com/blog/ small-business-matters/14-diy-mobile-app-development-resources-forsmall-businesses/2288. Accessed March 23, 2014.

49. The Office of the National Coordinator for Health Information Technology. About ONC. Available from: http://www.healthit.gov/ newsroom/about-onc. Accessed March 25, 2014.

50. US Department of Health and Human Services, Food and Drug Administration, Center for Devices and Radiological Health, Center for Biologics Evaluation and Research. Mobile medical applications: guidance for industry and food and drug administration staff; 2013. Available from: http://www.fda.gov/downloads/MedicalDevices/.../UCM263366.pdf. Accessed March 26, 2014.

51. USA Government. Mobile apps gallery; 2013. Available from: http:// apps.usa.gov/. Accessed March 23, 2014.

52. Hoffman B. Super Tracker yields super nutrition data. Food Tech Bytes; 2012. Available from: http://www.foodtechconnect.com/2012/01/18/ super-tracker-yields-super-nutrition-data/. Accessed March 23, 2014.

53. NIH mHealth Training Institute. NIH mHealth Training Institute. Conference 2-day workshop; 2014. Available from: http://www.mhealthsummit.org/program-details/nih-mhealth-training-institute. Accessed March 25, 2014.

54. Mobihealth. Alliances; 2007. Available from: http://www.mobihealth. com/profile/en/alliances.php\#. Accessed March 26, 2014.

55. Smith A. Smartphone ownership. Internet Project; 2013. Available from: http://pewinternet.org/Reports/2013/Smartphone-Ownership-2013. aspx. Accessed March 23, 2014

56. Tillmann K. Getting Connected with Emerging Fitness Technologies. 2012 mHealth Summit; December 3-5, 2012; National Harbor, MD.

57. Azar KM, Lesser LI, Laing BY, et al. Mobile applications for weight management: theory-based content analysis. Am J Prev Med. 2013;45(5):583-589.

58. Rastogi V, Chen Y, Enck W. Appsplayground: automatic security analysis of smartphone applications. Proceedings of the Third ACM Conference on Data and Application Security and Privacy; February 18-20, 2013; San Antonio, TX.

59. Su WC. A preliminary survey of knowledge discovery on smartphone applications (apps): principles, techniques and research directions for e-health. The 2014 International Conference on Complex Medical Engineering; June 26-29, 2014. Taipei, Taiwan. In press 2014.

60. Lightley D, Hughes R. A guide to healthcare and medical app development. Genetic Digital; 2014. Available from: http://www.geneticapps.co.uk/ white-papers/app-development/. Accessed March 26, 2014.

61. Cowart J. When to go native, mobile web or cross-platform/hybrid. TECHPRO; 2013. Available from: http://tech.pro/blog/1355/when-to-gonative-mobile-web-or-cross-platformhybrid. Accessed May 6, 2014.

62. Rabin C, Bock B. Desired features of smartphone applications promoting physical activity. Telemed J E Health. 2011;17(10):801-803.

63. Kranz M, Moller A, Hammerla N, et al. The mobile fitness coach: towards individualized skill assessment using personalized mobile devices. Perv Mobi Comp. 2013;9(2):203-215.

64. Hearn L, Miller M, Fletcher A. Online healthy lifestyle support in the perinatal period: what do women want and do they use it? Aust J Prim Health. 2013;19(4):313-318.

65. Jones BA, Madden GJ, Wengreen HJ. The FIT game: preliminary evaluation of a gamification approach to increasing fruit and vegetable consumption in school. 2014;pii:S0091-7435(14)00146-7. Epub April 2014.
66. Jones BA, Madden GJ, Wengreen HJ, Aguilar SS, Desjardins EA. Gamification of dietary decision-making in an elementary-school cafeteria. PLoS One. 2014;9(4):e93872.

67. National Association for Sport and PE (NASPE), Suzan Ayers, Mary Jo Sariscsany. Physical Education for Lifelong Fitness. Reston, VA: American Alliance for Health, Physical Education, Recreation and Dance; 1999:78-79.

68. Donnelly JE, Blair SN, Jakicic JM, Manore MM, Rankin JW, Smith BK; American College of Sports Medicine. American College of Sports Medicine Position Stand. Appropriate physical activity intervention strategies for weight loss and prevention of weight regain for adults. Med Sci Sports Exerc. 2009;41(2):459-471.

69. Silveira P, van de Langenberg R, van Het Reve E, Daniel F, Casati F, de Bruin ED. Tablet-based strength-balance training to motivate and improve adherence to exercise in independently living older people: a Phase II preclinical exploratory trial. J Med Internet Res. 2013;15(8): e159.

70. Kamel Boulos MN, Yang SP. Exergames for health and fitness: the roles of GPS and geosocial apps. Inter J Health Geogr. 2013; 12(18): $1-7$.

71. Currie D. Use of tools rises: more health departments nationwide embracing social media. The Nation's Health. 2012;42(4):1-18.

72. Kapp JM, LeMaster JW, Lyon MB, Zhang B, Hosokawa MC. Updating public health teaching methods in the era of social media. Public Health Rep. 2009;124(6):775-777.

73. Consumer Electronics Association. One-third of U.S. Consumers plan to purchase fitness technologies in 2013, according to CEA; 2012. Available from: http://www.ce.org/News/News-Releases/PressReleases/2012-Press-Releases/One-Third-of-U-S-Consumers-Plan-toPurchase-Fitne.aspx. Accessed March 31, 2014.

74. Bergman RJ, Spellman JW, Hall ME, Bergman SM. Is there a valid app for that? Validity of a free pedometer iphone application. $J$ Phys Act Health. 2012;9(5):670-676.

75. Health On the Net Foundation. The HON Code of Conduct for medical and health Web sites (HONcode). Available at: http://www.hon.ch/ HONcode/Webmasters/Conduct.html. Accessed March 25, 2014.

76. Plum Consulting and Vision Mobile. The European app economy: creating jobs and driving growth; 2013. Available at: http://www. visionmobile.com/product/the-european-app-economy/. Accessed June 4, 2014.

77. Powell AC, Landman AB, Bates DW. In search of a few good apps. JAMA. 2014;311(18):1851-1852.

78. KLAS. About KLAS. Available from: http://www.klasresearch.com/ about/company.aspx. Accessed March 25, 2014.

79. John D, Gunter K, Manore M, Langellotto G, Etuk L. Generating Rural Options for Weight Healthy Kids \& Communities. Poster presented at: the Society for Nutrition Education and Behavior Annual Conference; July 2012; Washington, DC. JNEB. 2012;44(4S):S84.

80. Johnston JD, Massey AP, Devaneaux CA. Innovation in weight loss programs: a 3-dimensional virtual-world approach. J Med Internet Res. 2012;14(5):e120

81. Napolitano MA, Hayes S, Russo G, Muresu D, Giordano A, Foster GD. Using avatars to model weight loss behaviors: participant attitudes and technology development. J Diabetes Sci Technol. 2013;7(4): 1057-1065.

82. Sullivan DK, Goetz JR, Gibson CA, et al. Improving weight maintenance using virtual reality (Second Life). J Nutr Educ Behav. 2013;45(3):264-268

83. Fox J, Bailenson J. Virtual self-modeling: the effects of vicarious reinforcement and identification on exercise behaviors. Media Psychology. 2009;12:1-25.

84. Mosa AS, Yoo I, Sheets L. A systematic review of healthcare applications for smartphones. BMC Med Inform Decis Mak. 2012;12:67. 
Smart Homecare Technology and TeleHealth

Dovepress

\section{Publish your work in this journal}

Smart Homecare Technology and TeleHealth is an international, peer-reviewed, open access online journal publishing original research, reviews, editorials and commentaries on the application of technology to support people and patients at home and in assisted living centers to optimize healthcare and management resources. Specific topics in the journal include: Development and application of

devices within the home and embedded in appliances; Healthcare provider communication and education tools; and drug ordering and adherence. The manuscript management system is completely online and includes a very quick and fair peer-review system, which is all easy to use. Visit http://www.dovepress.com/ testimonials.php to read real quotes from published authors.

Submit your manuscript here: http://www.dovepress.com/smart-homecare-technology-and-telehealth-journal 